\title{
RISCOS DO USO DE MEDICAMENTOS PARA O EMAGRECIMENTO
}

\author{
RISKS OF USING DRUGS FOR WEIGHT LOSS
}

\author{
Stephany Montenegro Lobo ${ }^{1}$ \\ Vicente Antonio de Senna Junior ${ }^{2}$ \\ Leonardo Guimarães de Andrade ${ }^{3}$
}

RESUMO: A obesidade é descrita como o acúmulo excessivo e anormal de gordura corporal e que em diferentes faixas etárias, a gordura atinge níveis que podem prejudicar a saúde. Sendo assim, para os pacientes que não conseguem obter perda de peso adequada, através de dietas, exercícios e alterações comportamentais, a terapia medicamentosa é mais indicada, juntamente com um monitoramento obtendo segurança e eficácia. Em síntese, fica claro que a maioria dos indivíduos preferem a utilização de fármacos anorexígenos, realizando assim a automedicação descontrolada. Dessa forma, a assistência farmacêutica é indispensável para orientar os usuários quanto à ação farmacológica desses medicamentos. Nessa pesquisa serão abordados os seguintes fármacos: Anfepramona, Fluoxetina e Sibutramina.

Palavras-chave: Inibidores de apetite. Obesidade. Emagrecimento. Riscos. Perda de peso.

ABSTRACT: Obesity is described as excessive and abnormal accumulation of body fat and, in different age groups, fat reaches levels that can harm health. Therefore, for patients who cannot achieve adequate weight loss through diets, exercises and behavioral changes, drug therapy is more indicated, along with monitoring obtaining safety and efficacy. In summary, it is clear that most individuals prefer the use of anorectic drugs, thus performing uncontrolled self-medication. Thus, pharmaceutical services are indispensable to guide users about the pharmacological

\footnotetext{
I Graduação em Farmácia na Universidade Iguaçu (UNIG). E-mail: stephanymlobo@gmail.com.

${ }^{2}$ Orientador. Mestre em Saúde Pública na Fundação Oswaldo Cruz (2005). Graduação em Ciências Biológicas na Universidade Gama Filho (1997) com especialização em Engenharia Sanitária e Controle Ambiental (2003) na Fundação Oswaldo Cruz, e em Gestão Ambiental na Universidade Federal do Rio de Janeiro (200o). Atualmente leciona como professor, nos cursos de Graduação de Farmácia, Enfermagem, Biologia, Nutrição e Radiologia na Universidade Iguaçu no Estado do Rio de Janeiro.. E-mail: vicente_sennajr@yahoo.com.br.

3 Co-autor. Mestre em Ciências do Meio Ambiente na Universidade Veiga de Almeida (2016). Graduação em Enfermagem na Universidade Iguaçu (2008) com Especialização em Enfermagem do Trabalho na Universidade Iguaçu (2009) e em Ensino e Biociência na Fundação Oswaldo Cruz (201I). Graduação em Odontologia com Especialização em Endodontia - ABO. Faz parte do corpo docente da Universidade Iguaçu no Estado do Rio de Janeiro. E-mail: leonard.gui@homail.com.
} 
action of these drugs. In this research, the following drugs will be addressed: Anfepramone, Fluoxetine and Sibutramine.

Keywords: Appetite suppressants. Obesity. Slimmin. Scratchs. Weight loss.

\section{INTRODUÇÃO}

De acordo com a definição da Organização Mundial de Saúde (OMS), a obesidade é descrita como o acúmulo excessivo e anormal de gordura corporal e que em diferentes faixas etárias, a gordura atinge níveis que podem prejudicar a saúde (LUCAS, et al., 202I). A obesidade em si, é um fator de risco para o bem-estar da população, pois além de afetar o desenvolvimento, também é uma causa influenciadora do surgimento de novas enfermidades (SANTOS, et al., 2019).

Desde a primeira pesquisa realizada em 1987 pelo Inquérito Nacional de Saúde, a obesidade tem aumentado em ambos os sexos, sendo mais evidentes nos homens do que nas mulheres. Além disso, há três fatores que influenciam diretamente esse processo: a idade do indivíduo obeso, o grau de obesidade e o tempo de evolução (SANTOS, et al., 2019).

No entanto, cientistas concluíram que a ingestão exagerada e o estilo de vida sedentário, são as principais razões pelo aumento da doença na sociedade ocidental. Embora que ainda existam informações nutricionais em escolas, clínicas e na internet, a obesidade continua sendo um grande problema (SANTOS, et al., 2019).

Diante disso, utentes buscam alternativas de emagrecimento mais rápidas. Portanto, as drogas anorexígenas são vistas como um recurso, porém, colocam em risco a saúde do usuário, gerando dependência, além de acarretar efeitos adversos quando ingeridas de formas descontroladas (ANDRADE, et al., 2019).

Segundo o Consenso Latino-Americano de Obesidade, quando as mudanças no estilo de vida não podem produzir o efeito desejado ou as comorbidades colocam em risco a vida de pacientes obesos, torna-se necessário a utilização da farmacoterapia. Como métodos utilizados no tratamento, podemos citar: a medicação não deve ser o único critério usado, devemos focar integralmente no tratamento com o paciente obeso, e não apenas na perda de peso, e o processo 
terapêutico deve ser prescrito e monitorado por especialistas (RADAELLI et al., 2016).

Por finalidade, o sucesso do tratamento de obesidade é considerado pela capacidade de atingir e manter uma perda de peso clinicamente útil, tendo resultados benéficos em relacionadas doenças, tais como diabetes mellitus tipo 2, hipertensão e dislipidemia. Um critério mínimo para o sucesso da perda de peso é de $5 \%$ contínuo, porque levará uma melhora pertinente à essas doenças. Visto que o sucesso a longo prazo dependerá de bastante atenção sobre a adequação nos níveis de atividades físicas, no controle da ingestão de alimentos e de outros fatores sendo apoios sociais, familiares e automonitoramento (MANCINI, 2016).

\section{OBJETIVO GERAL}

Analisar os agentes farmacológicos frequentemente utilizados no combate a obesidade, visando o esclarecimento de possíveis efeitos adversos e priorizando a conscientização dos benefícios e malefícios, através da observação dos respectivos mecanismos de ação de cada fármaco.

\section{OBJETIVOS ESPECÍFICOS}

- Conceituar o tratamento farmacológico na obesidade;

- Apresentar as classes dos respectivos fármacos: anfepramona, fluoxetina e sibutramina;

- Analisar os riscos e benefícios que cada fármaco pode ocasionar;

- Verificar quanto à ação farmacológica dos medicamentos anorexígenos;

- Conscientizar o público sobre as reações adversas da automedicação;

\section{METODOLOGIA}

Para a elaboração dessa pesquisa, realizou-se uma revisão literária onde foram feitos levantamentos bibliográficos por meio de artigos científicos, monografias, sites acadêmicos, revista SCIELO e em órgãos como Conselho Regional de Farmácia (CRF), Organização Mundial da Saúde (OMS) e Agência Nacional de Vigilância Sanitária (ANVISA), divulgados em um período entre 2009 a 202I. 
O objetivo dessa revisão de literatura é a busca pela atenção do farmacêutico, visando esclarecer os usuários dos medicamentos anorexígenos e seus malefícios, visto que, os utentes se automedicam sem auxílio do profissional, colocando assim sua saúde em risco.

\section{JUSTIFICATIVA}

A obesidade é uma doença crônica, onde muitas vezes os pacientes não conseguem perder o peso de uma forma rápida e eficiente sem auxílio de medicamentos, e com isso, buscam pela utilização de alguns fármacos inibidores de apetite sem nenhuma orientação profissional.

No entanto, essa pesquisa foi realizada com intuito de instruir sobre o funcionamento dos medicamentos no organismo, priorizando o entendimento do mecanismo de ação para que os usuários tenham consciência do verdadeiro objetivo dos fármacos, e assim reduzindo a utilização descontrolada dessa farmacoterapia.

\section{CONCEITO DO TRATAMENTO FARMACOLÓGICO PARA OBESIDADE}

Os medicamentos anorexígenos também conhecidos como inibidores de apetite, estavam no mercado há décadas, porém ainda enfrentam alguns problemas regulatórios, como a venda ilegal e o uso para outros fins, tornando-os vilões no tratamento da obesidade (ANDRADE, et al., 2019).

As drogas anorexígenas auxiliam na perda de peso reduzindo a fome, por isso, são chamadas de supressoras ou moduladoras de apetite, facilitando na saciedade dos usuários (OLIVEIRA, et al., 2009). "Estes fármacos atuam modulando neurotransmissões catecolaminérgicas e/ou serotoninérgicas" (OLIVEIRA, et al., 2009).

Os fármacos relativos à classe catecolaminérgica, agem ampliando a atividade de três neurotransmissores mediadas por catecolaminas tais quais, adrenalina, noradrenalina e dopamina, seja por inibir a recaptação ou por estimular a liberação de um ou mais neurotransmissores. Entre os fármacos referentes à essa classe, podemos destacar a anfepramona (OLIVEIRA, et al., 2009). 
$\mathrm{Na}$ classe serotoninérgica estão presentes os fármacos fluoxetina e sibutramina, devido à ativação dos receptores da serotonina e norepinefrina, agem promovendo a sensação de saciedade (OLIVEIRA, et al., 2009). Segundo algumas pesquisas farmacológicas, os serotoninérgicos são capazes de controlar o peso corporal, sendo assim, a serotonina é conhecida por seu efeito regulador sobre ingestão de alimentos (GARCIA, 2017 apud LUCAS, et al., 2021).

Logo, a anfepramona e a sibutramina são medicamentos controlados especiais, sendo retidos e vendidos com receita e prescrição médica. No entanto, o alto consumo no Brasil revela que suas indicações clínicas e o acesso a esses fármacos não estão de acordo com as recomendações da Organização Mundial da Saúde (OMS) e de órgãos sanitários, consequentemente acarretando o seu uso indevido (BRASIL, 20II apud DUARTE, et al., 2020).

Sendo assim, para os pacientes que não conseguem obter perda de peso adequada após 6 meses de mudanças na dieta, exercícios e alterações comportamentais, a terapia medicamentosa é mais indicada, juntamente com um monitoramento obtendo segurança e eficácia (KARIN, 20I8).

\section{BENEFÍCIOS E RISCOS DOS FÁRMACOS}

\section{ANFEPRAMONA}

Inicialmente, a anfepramona foi desenvolvida para o controle de crianças hipercinéticas, auxiliando também no tratamento de narcolepsia (RADAELLI, et al., 2016). Além disso, assemelha-se a anfetamina pois possuem estruturas parecidas (RADAELLI, et al., 2016).

No entanto, foi observado que os indivíduos que utilizavam esse medicamento, obtinham uma redução na fome. Com isso, passou a ser conhecida como fármaco anorexígeno. Ainda que a eficácia seja rápida até 20 semanas, verificou-se que não há respostas à longo prazo, por questões de tolerância desenvolvida pelo organismo, pois o fármaco acaba perdendo o efeito desejado (BRASIL, 20II apud DUARTE, et al., 2020).

Além disso, as administrações feitas da anfepramona de doses terapêuticas variam entre $50 \mathrm{mg}$ a $100 \mathrm{mg}$ do medicamento, porém, não trouxeram eficácia e 
segurança no tratamento com doses elevadas, apesar disso, a dosagem mais utilizada do fármaco é de $25 \mathrm{mg}$ com o tempo de meia vida de 4 a 6 horas (RADOMINSKI, et al., 2010).

De acordo com Naccarato e colaboradores (p. 68, 2014) o mecanismo de ação da anfepramona:

Atua na fenda sináptica inibindo a recaptação de noradrenalina e aumentando a interação desse neurotransmissor com receptores póssinápticos, nos centros da alimentação e saciedade do hipotálamo, diminuindo a fome. Em testes realizados com animais, demonstrou ação redutora na ingestão de alimentos. Contudo, o aumento da atividade noradrenérgica não é seletivo, resultando em efeitos adrenérgicos periféricos.

Segundo a Anvisa (20II) "anfepramona pode promover reações adversas graves, tais como agranulocitose, arritmia cardíaca, isquemia cerebral, acidente cerebrovascular, dependência, leucemia, hipertensão pulmonar primária e distúrbios psicóticos” (DRUGDEX, 20II apud ANVISA, 20II).

\section{FLUOXETINA}

É um medicamento antidepressivo, podendo auxiliar no emagrecimento, pois um dos seus efeitos adversos é a perda de peso. Apesar disso, esse fármaco deve ser utilizado somente sob prescrição médica, pois ainda que auxilie no emagrecimento, o mesmo pode acarretar efeito rebote, fazendo com que o usuário ganhe ainda mais peso (SANTOS, et al., 2019). Contudo, sua farmacoterapia em relação a obesidade é caracterizada de forma off label, este termo refere-se ao uso de um determinado medicamento com um propósito distinto do que é indicado (TANEMURA, 2019 apud LUCAS, et al., 202I).

Segundo Karin (p.4, 2018) "Fluoxetina é um agente serotoninérgico que tem sido prescrito para perda de peso, apesar de seu mecanismo de ação na obesidade ser desconhecido". Embora seu efeito aconteça por meio da inibição seletiva de recaptação da serotonina, aumentará cada vez mais a neurotransmissão. Visto que seu metabolismo é determinado como farmacologicamente ativo, tendo um tempo de ação prolongado (LOPES, 2015). 
Apesar disso, os inibidores seletivos da recaptação da serotonina reduzem o metabolismo das isoenzimas do citocromo P45o (CYP) de forma dose-dependente, o que significa que o aumento na dose de administração de fluoxetina levará a níveis plasmáticos, meia-vida e possíveis efeitos colaterais. Com isso, será essencial um período para alcançar o estado de equilíbrio no organismo (ROCHA, et al., 2013).

Em pesquisas, foram observados que algumas reações como convulsões, aumento do risco de quedas de pressão arterial, sangramento gastrointestinal e doenças relacionadas ao controle do diabetes têm sido relatadas como efeitos adversos relacionadas ao uso de fluoxetina (DENNIS et al., 2019 apud, LUCAS et al., 2021). Além das reações citadas anteriormente, também são descritos sintomas como distúrbios do sono, redução da libido, amnésia, xerostomia, sudorese, cefaleia e dificuldade na coordenação motora (LOPES, 2015).

\section{SIBUTRAMINA}

A princípio, a sibutramina foi desenvolvida com a função de fármacos antidepressivos, porém, entre estudos clínicos foi observado uma ineficácia no tratamento da depressão, entretanto, este medicamento era eficiente na perda de peso, logo, passou a ser utilizado como inibidor de apetite (ANDRADE, et al., 2019).

A sibutramina tem o efeito de causar a saciedade, ou seja, não tem a função de reduzir o apetite, com isso, pacientes que utilizaram essa droga acabam se alimentando menos, pois se saciam mais rápido (DUTRA, et al., 2015).

Entretanto, este fármaco é rapidamente absorvido pelo trato gastrointestinal após a administração via oral. Sofrendo uma metabolização de primeira passagem pelo citocromo $\mathrm{P}_{450}$ no fígado. Além disso, a concentração máxima pode alcançar entre 3 a 4 horas, com meia-vida de 14 a 16 horas. Desta forma, a principal via de eliminação será nos rins (MANCINI, 2016). Com isso, a administração desse medicamento é realizada em doses terapêuticas de $10 \mathrm{mg}$ a $15 \mathrm{mg}$, e a dosagem máxima sendo de $20 \mathrm{mg}$, porém não houveram resultados de eficácia e segurança em doses elevadas (RADOMINSKI, et al., 2010).

No entanto, o uso prolongado da sibutramina estimula a perda de peso pelo aumento metabólico, por meio da redução do gasto de energia de repouso. Visto que 
a utilização desse fármaco é questionável em pacientes obesos e com hipertensão controlada (ANDRADE, et al., 2019).

Deste modo, a sibutramina é uma classe de inibidores da recaptação da noradrenalina e serotonina ( 5 - Hidroxitriptamina; 5-HT). Esta droga bloqueia a noradrenalina pré-sináptica e os receptores 5 - $\mathrm{HT}$ nos centros hipotalâmicos de alimentação e saciedade, potencializando os efeitos anorexígenos dos neurotransmissores do sistema nervoso central (SNC), obtendo-se uma redução da fome. Sendo assim, os efeitos envolvidos pela saciedade são relacionados à serotonina 5-HT2c. Além disso, a noradrenalina e a serotonina desempenham um papel fundamental no gerenciamento das emoções das pessoas, onde estão relacionadas o controle da obesidade, depressão e ansiedade (ANDRADE, et al., 2019).

No entanto, esse fármaco se sobressai em relação aos demais inibidores de apetite, pelo fato de aumentar a liberação de serotonina e noradrenalina. Além do mais, não inibem a recaptação e nem aumentam a liberação de dopamina como agem nos outros medicamentos (ANDRADE, et al., 2019).

Após a administração da sibutramina, a frequência cardíaca e a pressão arterial acabam sendo estimuladas, aumentando os batimentos aproximadamente em 5 bpm (batimentos cardíacos por minuto) e a pressão arterial chega a cerca de 3 a 4 mmHg (milímetros de mercúrio). Com isso, ocorre uma redução na pressão arterial na maior parte dos utentes. Através de diversos testes clínicos, este fármaco promoveu um declínio estatisticamente significativo no peso corporal, a partir de concentração de colesterol total, triglicerídeos, colesterol de lipoproteína de baixa densidade e hemoglobina glicada de pacientes obesos, diabéticos e não obesos (ANDRADE, et al., 2019).

As reações adversas mais comuns são, dores de cabeça, boca seca, prisão de ventre, insônia e hipertensão. Os efeitos colaterais ocorrem em 10\% a 20\% dos casos. Contudo, os efeitos adicionais adrenérgicos induzidos pela sibutramina podem aumentar a pressão arterial em pacientes obesos. Sintomas como irritabilidade, ansiedade, náuseas e taquicardia são menos recorrentes. Por isso, não há evidências 
de que a sibutramina cause anomalias valvulares ou hipertensão pulmonar (RADAELLI, et al., 2016).

No quadro a seguir, são apresentados os medicamentos mais utilizados no tratamento da obesidade, mostrando a dosagem utilizada, sua dependência e o efeito de cada fármaco.

Quadro r: Medicamentos mais utilizados para o emagrecimento.

\begin{tabular}{|l|l|l|l|}
\hline Medicamentos & Anfepramona & Fluoxetina & Sibutramina \\
\hline Dosagem & 40 a $20 \mathrm{mg} / \mathrm{dia}$ & $60 \mathrm{mg} / \mathrm{dia}$ & Io a $20 \mathrm{mg} / \mathrm{dia}$ \\
\hline Dependência & IV & Baixa & Baixa \\
\hline Efeito dos fármacos & Diminuição da fome. & Antidepressivo, & Aumenta \\
& & aumenta a saciedade. & saciedade. \\
\hline
\end{tabular}

Fonte: Adaptado por LOBO (2021).

O grau de dependência mencionado em cada fármaco é fundamental, pois a capacidade de induzir a dependência desses medicamentos varia de I a IV (MUER, 2015). Porém, no caso da anfepramona, se administrado em grandes quantidades, o paciente torna-se dependente do medicamento. Portanto, a anfepramona é adequada para o tratamento à curto prazo, com efeito rápido e eficaz de até 20 semanas. (RADAELLI, Et Al., 2or6).

\section{DISCUSSÃO}

Perante o que foi exposto, percebe-se que os indivíduos preferem em grande parte dos casos, a utilização de fármacos inibidores de apetite, em consequência de não conseguirem uma perda de peso naturalmente. Porém, o fato de automedicaremse sem auxílio de um profissional, é o principal entre os diversos fatores que acarretam risco à saúde desses indivíduos.

Em consideração a isso, o farmacêutico é quem informa e tem o dever de alertar sobre os perigos que os fármacos podem ocasionar no organismo, e ainda 
esclarecer que a utilização desses medicamentos para fins estéticos não é o caminho mais apropriado e seguro.

Por outro lado, apesar de todas as contraindicações, os medicamentos na maioria das vezes é a única opção para pacientes obesos ou com sobrepeso, visto que os mesmos possuem mais dificuldades para alcançarem o emagrecimento seja por genética, condicionamento físico ou até mesmo contratempos para manterem um ritmo de vida saudável.

Por fim, é de suma importância ressaltar que além de uma melhora ao bemestar, o emagrecimento também previne possíveis futuras doenças relacionadas ao excesso de peso.

\section{CONCLUSÃO}

Em suma, a terapia medicamentosa de forma contida mostrou-se favorável, deste modo, os utentes conquistaram resultados positivos obtendo uma perda de peso gradual, proporcionando assim, uma saúde balanceada e consequentemente, provocando o aumento da autoestima. No entanto, como cada medicamento possui contraindicações, é necessário que o farmacêutico enfatize os efeitos adversos associados quanto ao uso contínuo daquele determinado fármaco ao prescrevê-lo.

Desta forma, a assistência farmacêutica é imprescindível com objetivo de auxiliar os usuários no entendimento da ação farmacológica dos medicamentos anorexígenos, a fim de proporcionar um tratamento satisfatório e cauteloso.

\section{REFERÊNCIAS BIBLIOGRÁFICAS}

ANDRADE, T. B. et al; O farmacêutico frente aos riscos do uso de inibidores de apetite: A Sibutramina. Revista Científica da Faculdade de Educação e Meio Ambiente, Rondônia, v. Io, n. I, p. 81-92, jul. 2019.

BRASIL, Agência Nacional de Vigilância Sanitária-ANVISA. Nota técnica sobre eficácia e segurança dos medicamentos inibidores de apetite. Brasília: Ministério da Saúde, p. 86, 20II.

DUARTE, A. P. N. B. et al; Uso de anfepramona, femproporex, mazindol, e sibutramina no tratamento de pacientes com sobrepeso ou obesidade: Análise 
farmacológica e clínica. Universidade Federal de São Paulo, São Paulo, v. 6, n. 2, p. I8,2020 .

DUTRA, J. R. et al; A influência dos padrões de beleza vinculados pela mídia, como fator decisório na automedicação com moderadores de apetite por mulheres no município de Miracema-Rj. Revista Transformar, v. 9, n.7, p.194-213, 2015.

KARIN, J. B. Z. et al; O uso off label de medicamentos para obesidade. Centro de Informação sobre Medicamentos do Conselho Regional de Farmácia, Pará, n. 2, jun. 2018.

LOPES, D. D. S; O consumo de psicoativos: análise da psicofarmacologia de antidepressivos. Revista Científica Facider, Mato Grosso, v. 7, n. 7, p.I-19, 2015.

LUCAS, B. B. et al; Farmacoterapia da obesidade: Uma revisão de literatura. Revista UEPB, Universidade Federal de Campinas Grande, Centro de Educação e Saúde, Paraíba, v. I7, n. I, jan. 2021.

MANCINI, M. C.; Obesidade e sobrepeso: tratamento farmacológico. Revista Diretrizes Brasileiras de Obesidade, São Paulo, v. 46, n. 5, p. 55- 66, 2016.

MURER, E; Drogas e remédios para emagrecer. Unicamp, São Paulo, ago. 2015.

NACCARATO, M. C. et al; Uso dos anorexígeneo anfepramona e sibutramina: benefícios ou prejuízo à saúde?. Revista Saúde, São Paulo, v. 8, n. I/2, p. 66-72, 2014.

OLIVEIRA. R. C. et al; A farmacoterapia no tratamento da obesidade. Revista Brasileira de Obesidade, Nutrição e Emagrecimento, São Paulo, v. 3, n. 17, p. 375-388, set./out. 2009.

RADAELLI, M. et al; Farmacoterapia da obesidade: Benefícios e Riscos. Revista Saúde e Desenvolvimento Humano, Rio Grande do Sul, v. 4, n. I, abr. 2016.

RADOMINSKI, R. B. et al; Atualização das diretrizes para o tratamento farmacológico da obesidade e do sobrepeso. Revista Diretrizes Abeso, v. 4, n. 76, p. II5, out. 2010.

ROCHA, B. S. et al; Psicofármacos na estratégia saúde da família: perfil de utilização, acesso e estratégia para a promoção do uso racional. Revista Ciência Saúde Coletiva, v. I8, n. II, p. 3291-3300, jul. 2013.

SANTOS, K. P. et al; Perigo dos medicamentos para emagrecer. Revista de Iniciação Científica e Extensão, Goiás, v. 2, n. I, p. 37-45, dez. 2018. 